\title{
On the Recovery of Shape and Reflectance from a Single Multispectral Image
}

\author{
Sejuti Rahman ${ }^{1} \quad$ Antonio Robles-Kelly ${ }^{1,2}$ \\ ${ }^{1}$ Research School of Engineering, Australian National University, Canberra ACT 0200, Australia \\ ${ }^{2}$ National ICT Australia (NICTA), , Locked Bag 8001, Canberra ACT 2601, Australia
}

\begin{abstract}
This paper presents a method for recovering the reflectance and shape of a surface from a single hyperspectral image. To do this, we depart from a reflectance model based on a physical interpretation of the reflection process where the geometry of the scene and the properties of the object surface describe the image formation process. With the model in hand, we propose a solution to the recovery of the reflection parameters via an optimisation approach that aims at recovering both the surface normals and the reflectance. This, in turn, allows for the application of the method by Frankot and Chellappa [8] so as to recover the surface depth at each pixel. We demonstrate the utility of our method for the recovery of both reflectance and surface depth on synthetic and real world images.
\end{abstract}

\section{Introduction}

Photometric invariants are an important research topic in computer vision which offers great opportunities in a variety of tasks such as material and object identification, recognition and pose estimation. With the advent of multispectral imaging, it is possible to represent the surface radiance over thousands of bands across a broad spectrum. This ability to represent illumination and surface reflectance as a spectral signature opens up the opportunity to develop photometric invariants which are both, high-dimensional and allow for the recovery of shape information from single views.

In computer vision, there has been considerable research on developing invariants for recognition. For instance, Belhumeur and Kriegman [3] have proposed a method for recognition which hinges in the recovery of a low dimensional linear subspace for imagery taken under a variety of lighting conditions. Nayar and Bolle [23] have used a reflectance ratio for object recognition. Dror et al. [6] have proposed a method for classifying objects based on their reflectance properties. Narasimhan et al. [21] have derived

\footnotetext{
*National ICT Australia is funded by the Australian Government's Backing Australia's Ability initiative, in part through the Australian Research Council.
}

a new class of photometric invariants where the scene radiance can be decomposed into material related terms and object shape and lighting. Despite being effective, these methods often assume Lambertian surfaces [3] or a specific surface geometry [29]. Other methods elsewhere in the literature resort to specific hardware [23], colour segmentation as a preprocessing step [13] or impose constraints on the input imagery [19].

The intrinsic relation between photometric invariance and shape recovery has also been widely studied in the community. This is because the reflectance of an object does not depend only on the light source and viewing directions, but also on material properties of the surface under study and its shape. This is closely related to the problem of recovering the shape of an object from its shading information. Shape-from-shading is concerned with recovering local estimates of the surface normal direction, and hence the surface height function, from single images of shaded surfaces. The problem is a classical one which has been studied in both the computer vision and photogrammetry literature for a number of decades. In photogrammetric remote sensing the problem is known as photoclinometry, and one of the earliest practical applications was to recover the shapes of lunar maria from optical images [26]. More recent work has extended the technique to the radar domain and has developed radar-clinometry methods to analyse the topography of the surface of Venus using images delivered by the Magellan probe [8]. Remote sensing has also lead to the development of the technique referred to as photometric stereo, which involves surface normal and height recovery from sets of images recovered under different light source directions [34].

In computer vision, some of the first shape from shading work was reported in the PhD theses by Horn [9] and by Krakauer [14]. The analysis of the literature on the topic of surface height recovery is not a straightforward task. The reason for this is that surface recovery is frequently viewed as an integral part of the shape-from shading or shape-fromtexture process. Horn and Brooks [11, 10] realise surface height recovery as a post-processing step. The process proceeds from the occluding boundary and involves incre- 
menting the surface height by an amount determined by the distance traversed and the slope angle of the local tangent plane. In some of the earliest work, $\mathrm{Wu}$ and $\mathrm{Li}$ [36] average the surface normal directions to obtain a height estimate. A more elegant solution is proposed by Frankot and Chellappa [8] who project the surface normals into the Fourier domain to impose integrability constraints whereby surface height is recovered using an inverse Fourier transform. Klette and his co-workers have enhanced this approach by showing how more complex regularisation constraints can be formulated in the Fourier domain [31]. Leclerc and Bobick [16] have developed a direct numerical method for height recovery from shading information which uses curvature consistency constraints. Dupuis and Oliensis [7] have developed a method which draws on differential geometry and involves propagation in the direction of the steepest gradient from singular points. A fast variant of this algorithm is described by Bichsel and Pentland [4] who compute the relative height of the surface with respect to the highest intensity point. A review and comparative study of a number of the methods above is presented in [37].

By contrast, in computer graphics, it is the development of computationally efficient tools for the purposes of realistic surface rendering that is of primary interest, and, hence, it is empirical models that have been the focus of activity $[5,25]$. One of the most popular models is that developed by Phong [25]. Torrance and Sparrow [30] have developed a more physically realistic account of specular reflectance that models the angular distribution of surface microfacets. Lewis [18] has also derived a more physically plausible variant of the Phong model. Additionally, Schlick [27] has presented a useful numerical simplification of Phong's model. A survey of reflectance and shading models can be found in [28].

However, neither the models developed in physics nor the computational models developed in graphics are well suited for surface analysis tasks in computer vision. It is for this reason that Wolff [33] and Nayar and Oren [22] have developed phenomenological, or semi-empirical, models that account for departures from Lambertian reflectance.

In this paper, we present a method to recover both, the reflectance and the object shape from a single multipsectral or hyperspectral image. The paper is organised as follows. In Section 2, we present the reflectance model used throughout the paper. Section 3 presents the optimization approach along with cost functions and constraints used to recover photometric parameters. Section 4 presents experimental results and analysis for both synthetic and real world images. Finally, conclusions are given in Section 5.

\section{Photometric Invariant Recovery}

As mentioned earlier, a number of reflectance models have been proposed so as to account for a number of pho-

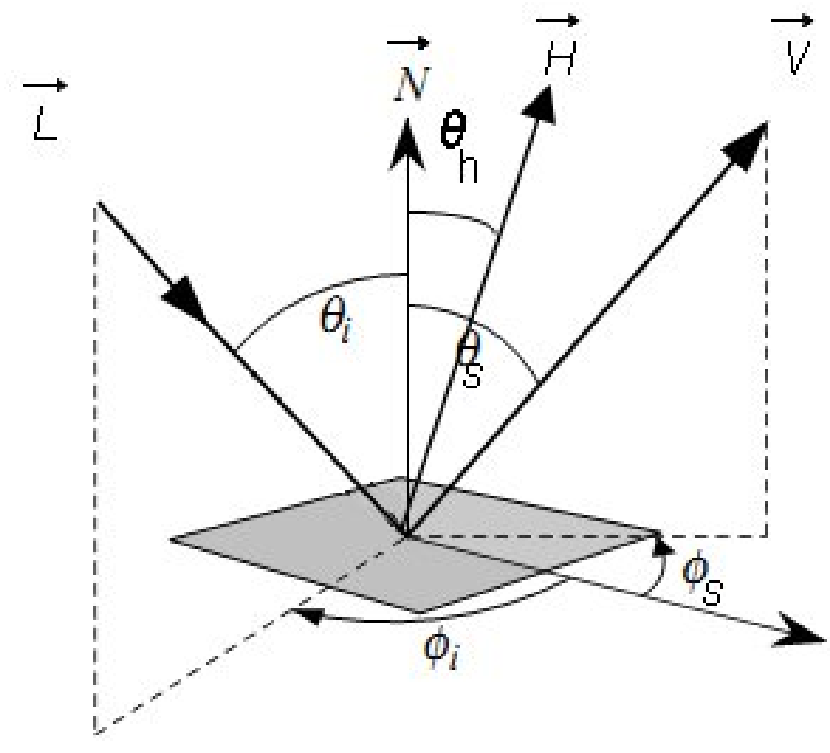

Figure 1. The geometry of reflection

togrammetric phenomena. Here, we use a linear combination of existing models where each term represents the specular lobe and diffuse reflectance subject to wavelength constraints. We have selected the Torrance-Sparrow model [30] to represent specular lobe of the reflection process. Nayar et al. [24] have shown that, though this model can not be used to describe the specular spike, it is able to approximate specular lobe of smooth surfaces. Therefore, for both smooth and rough surfaces, we use the Torrance-Sparrow model to model the specular lobe. To represent the specular spike for smooth surfaces, we employ the Beckmann-Kirchhoff model [2]. For diffuse reflectance, we use Wolff's model [33]. The linear combination of these models is, therefore, given by

$$
f(u, \lambda)=W_{s}\left(f_{T S}(u, \lambda)+f_{B K}(u, \lambda)\right)+W_{d} f_{w}(u, \lambda)
$$

where, $f_{T S}, f_{B K}, f_{w}$ denote the reflectance equation of Torrance-Sparrow, Beckmann-Kirchhoff and Wolff respectively, $W_{s}$ denotes the weight for the specular component and $W_{d}$ corresponds to the contribution of the diffuse component.

In the following subsections we ellaborate further on these terms by presenting the models and their variables. It is worth noting that all these models herein follow the reflection geometry given in Figure 1 . In the figure, $\vec{N}$ is the unit surface normal, $\vec{L}$ is the unit incident vector with zenith angle $\theta_{i}$ and azimuth angle $\phi_{i}$ and $\vec{V}$ is the unit view vector defined by zenith angle $\theta_{s}$ and azimuth angle $\phi_{s}$. The half-way vector is denoted by $\vec{H}$ and is given by the angular bisector of $\vec{L}$ and $\vec{V}$, i.e. $\vec{H}=\vec{L}+\vec{V}$. We denote the angle between $\vec{N}$ and $\vec{H}$ as $\theta_{h}$. 


\subsection{Torrance-Sparrow Model}

As mentioned earlier, the Torrance-Sparrow model describes the specular reflection on rough surfaces. The main idea is to model a surface as a collection of perfectly smooth mirror-like microfacets. The corresponding reflectance equation has both diffuse and specular reflectance terms. Here, we use only the specular component of the model given by

$$
f_{T S}(u, \lambda)=\frac{A_{f}}{4} F\left(\theta_{i}, \eta(\lambda)\right) \frac{G\left(\theta_{i}, \theta_{s}, \phi_{s}\right)}{\cos \theta_{s}} P_{\alpha}(\alpha)
$$

where,

$G\left(\theta_{i}, \theta_{s}, \phi_{s}\right)=\min \left(1, \frac{2(\vec{N} \cdot \vec{H})(\vec{N} \cdot \vec{V})}{(\vec{V} \cdot \vec{H})}, \frac{2(\vec{N} \cdot \vec{H})(\vec{N} \cdot \vec{L})}{(\vec{V} \cdot \vec{H})}\right)$

The specular reflectance component consists of microfacet area $A_{f}$, Fresnel term $F(\cdot)$, geometrical attenuation factor $G$ and microfacet slope distribution function $P_{\alpha}$. The Fresnel term $F(\cdot)$ determines the fraction of incident light that is reflected by each facet, whereas $G$ accounts for masking and shadowing of one microfacet by adjacent facets.

In the model, $P_{\alpha}$ is a microfacet distribution function. Consider an infinitesimal surface patch which has mean surface orientation $\vec{N}$. Each constituent microfacet of this patch has its own orientation, which deviates from $\vec{N}$ by an angle $\alpha$. The facet slope distribution function $P_{\alpha}(\alpha)$ used by the model is a normal one with zero mean, i.e. $\bar{\alpha}=0$, and standard deviation $\sigma_{\alpha}$. The distribution is rotationally symmetric about the mean surface normal, $\vec{N}$, hence, we have

$$
P_{\alpha}(\alpha)=\frac{1}{\sqrt{2 \pi} \sigma_{\alpha}} \exp \left(-\frac{\alpha^{2}}{2 \sigma_{\alpha}^{2}}\right)
$$

where $\sigma_{\alpha}$ is the so-called roughness parameter.

\subsection{Beckmann-Kirchhoff Model}

The Beckmann- Kirchhoff model describes the reflection from surface as a sum of two terms

$$
P\left(\theta_{i}, \theta_{s}, \phi_{i}, \phi_{s}\right)=P_{0}^{2} \exp \left(-g\left(\theta_{i}, \theta_{s}\right)\right)+D\left(\theta_{i}, \theta_{s}, \phi_{i}, \phi_{s}\right)
$$

The former denotes the scattering component in the specular direction and the latter represents the diffuse scattering. In this paper, we are interested in the first term. The specular spike from a surface whose height $h$ is normally distributed, as shown in (8), is given by:

$$
f_{B K}=P_{0}^{2} \exp \left(-g\left(\theta_{i}, \theta_{s}\right)\right)
$$

where,

$$
g=\left(2 \pi \frac{\sigma_{h}}{\lambda}\left(\cos \theta_{i}+\cos \theta_{s}\right)\right)^{2}
$$

Here, $g$ represents the surface roughness, where $P_{0}$ is a function of $\theta_{i}$ and $\theta_{s}$ that increases rapidly about the specular direction. In the Beckmann-Kirchhoff model, $P_{0}$ is expressed as a sinc function. Here, following Nayar et al. [24], we use a Gaussian function with a very small standard deviation.

Note that the Beckmann-Kirchhoff model considers a normal distribution to express the uncertainity in height. According to this model, the height distribution function of a surface with mean value $\bar{h}=0$, standard deviation $\sigma_{h}$, and correlation distance $\mathrm{T}$, is given by

$$
P_{h}(h)=\frac{1}{\sqrt{2 \pi} \sigma_{h}} \exp \left(-\frac{h^{2}}{2 \sigma_{h}^{2}}\right)
$$

where $\sigma_{h}$ is the mean square root of $h$ and represents the roughness of a surface.

\subsection{Wolff Model}

Wolff [32] has proposed a diffuse reflectance model for smooth dielectric surfaces. Note that, when the angle between illuminant and viewing directions is large, significant deviation from Lambert's law [15] is prevalent for many dielectric objects. The Wolff model explains this deviation from Lambertian reflaction by considering refractive attenuation at the surface-air layer. It multiplies Lambert's cosine law by two Fresnel terms: one for incident light and the other for reflected light. The diffuse reflectance, as defined by the Wolff model is given by:

$$
f_{w}=\rho \cos \theta_{i}\left[1-F\left(\theta_{i}, \eta(\lambda)\right)\right]\left[1-F\left(\theta_{s}^{\prime}, \frac{1}{\eta(\lambda)}\right)\right]
$$

Here, $\rho$ is the total diffuse albedo, $\eta$ is the index of refraction of the dielectric medium, $\theta_{i}$ is the external angle of incidence, and $\theta_{s}^{\prime}$ is the internal angel of incidence that relates to reflected angle $\theta_{s}$ by Snell's law, i.e. $\theta_{s}^{\prime}=\arcsin \left(\frac{\sin \left(\theta_{s}\right)}{\eta(\lambda)}\right)$.

\section{Optimisation}

Our aim is to estimate reflectance model parameters given by the diffuse albedo $\rho$ and surface normal $\vec{N}$ by fitting the linear combination in Equation 1 to the image radiance $R\left(v, \lambda_{i}\right)$ at pixel $v$ and band $\lambda_{i}$. To this end, we use the Levenberg-Marquardt algorithm [17, 20] for purposes of minimising the cost functions

$$
\begin{gathered}
C_{\rho\left(u, \lambda_{i}\right)}=\sum_{v \in \wp}\left(\left(R\left(v, \lambda_{i}\right)-f\left(\rho\left(v, \lambda_{i}\right), \lambda_{i}\right)\right)^{2}+\right. \\
\left.\sum_{v \in \wp}\left(\rho\left(u, \lambda_{i}\right)-\rho\left(v, \lambda_{i}\right)\right)^{2}\right) \\
C_{\vec{N}}(u)=\sum_{i \in w}\left(\left(R\left(u, \lambda_{i}\right)-f\left(\vec{N}(u), \lambda_{i}\right)\right)^{2}\right)
\end{gathered}
$$



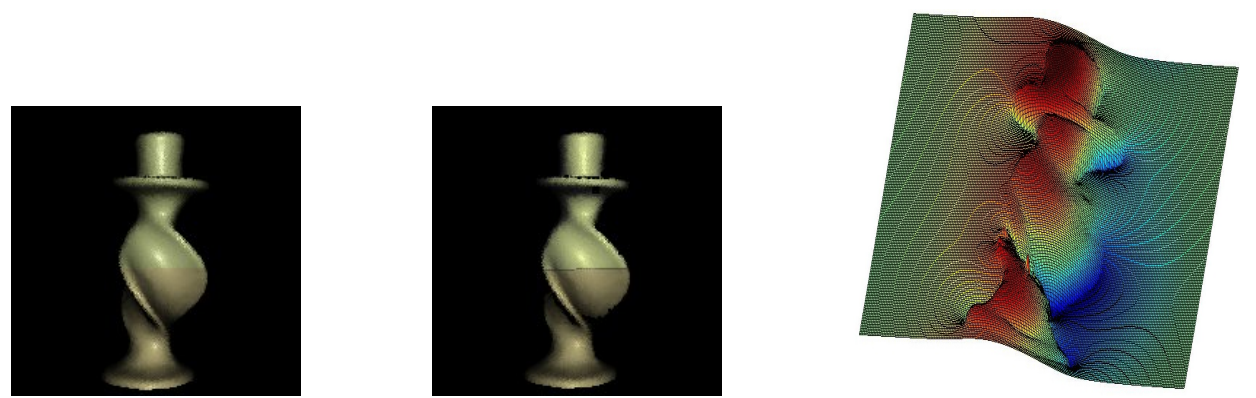

Figure 2. Results for a synthetic image. From left to right: (a) Input image; (b) Estimated image rendered with computed parameters; (c) Depth field
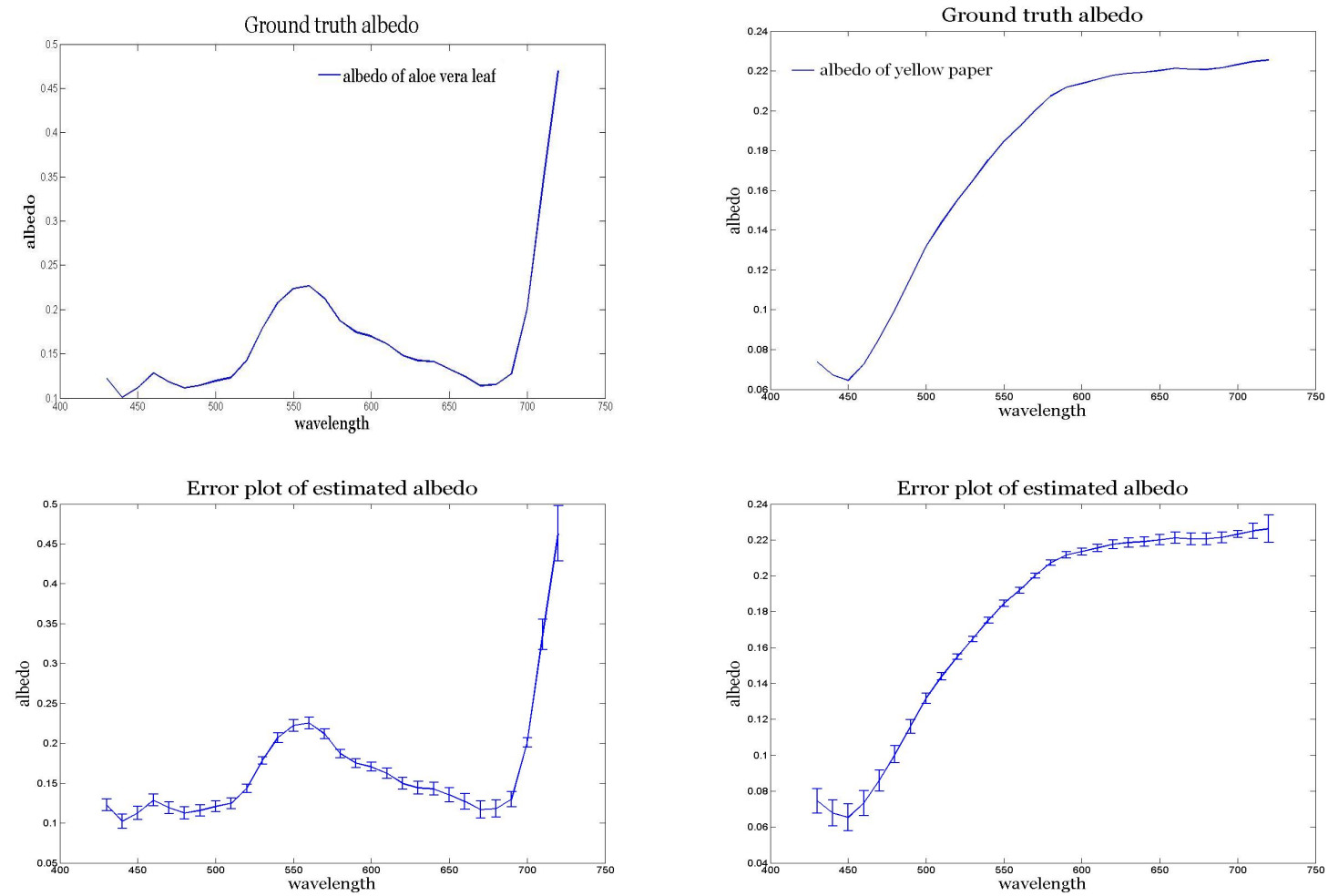

Figure 3. From left-to-right and top-to-bottom, (a) The plot of the ground truth albedo used for the k1 region (upper part) of the candle stick; (b) The plot for the ground truth albedo used for the k2 region (lower part) of candle stick; (c) The plot of the average estimated albedo for all pixels in $\mathrm{k} 1$ region; (d) The plot of average estimated albedo for all pixels in $\mathrm{k} 2$ region.

Note that the cost function in Equation 10 is constrained over the neighbourhood $\wp$ centered at the pixel under study. The cost function for $\vec{N}$ at pixel $u$ is given in Equation 11 .

Our optimisation scheme takes at input a single hyperspectral image whose bands are indexed to wavelength $\lambda \in\left\{\lambda_{1}, \ldots \ldots, \lambda_{l}\right\}$. The image $I(u, \lambda)$ is comprised of the spectral radiance at location $u$ and wavelength $\lambda$. The illuminant direction is unknown and the viewer's position is fixed. For any location $u$, the $x-y$ coordinates correspond to row and column, respectively, where the surface depth $z$ is initialized by any arbitrary value. At each iteration, $z$ is updated using the method in [8]. As a pre-processing step, we compute the illumination power spectrum $L(\lambda)$ using [12] so as to recover the spectral reflectance $R(u, \lambda)=\frac{I(u, \lambda)}{L(\lambda)}$. We use Worthington and Hancock's robust regularisation method [35] to compute the initial estimate of the surface normals. The algorithm first solves for $\rho\left(u, \lambda_{i}\right)$ using Equation (10). Based on the estimated reflectance $\rho$, we recover the surface normals $\vec{N}$ making use of Equation (11). 

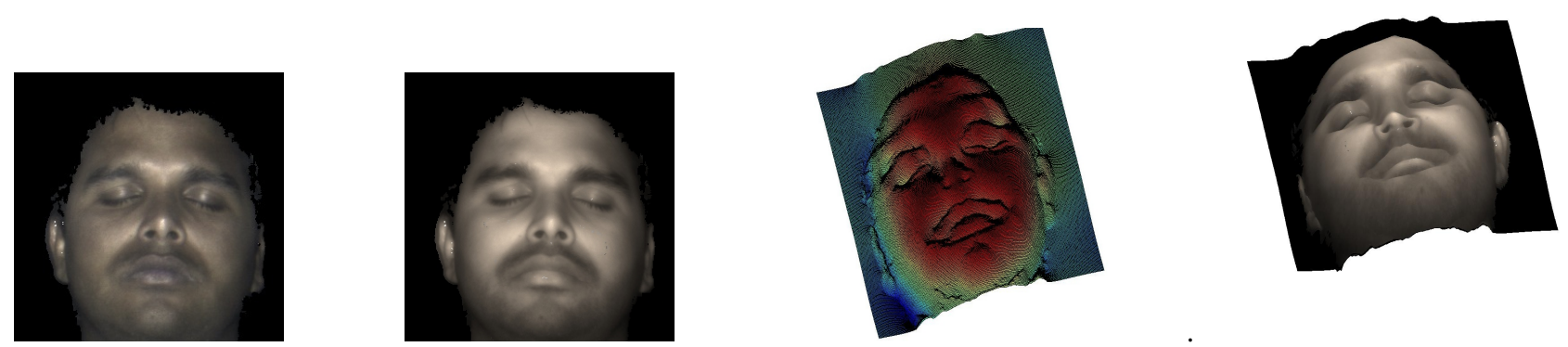

Figure 4. Results for a real world image. From left to right: The input image; Estimated image rendered with the recovered surface normals and albedo; Depth map; Result of texture-mapping the image in the second column onto the depth map in the third column.
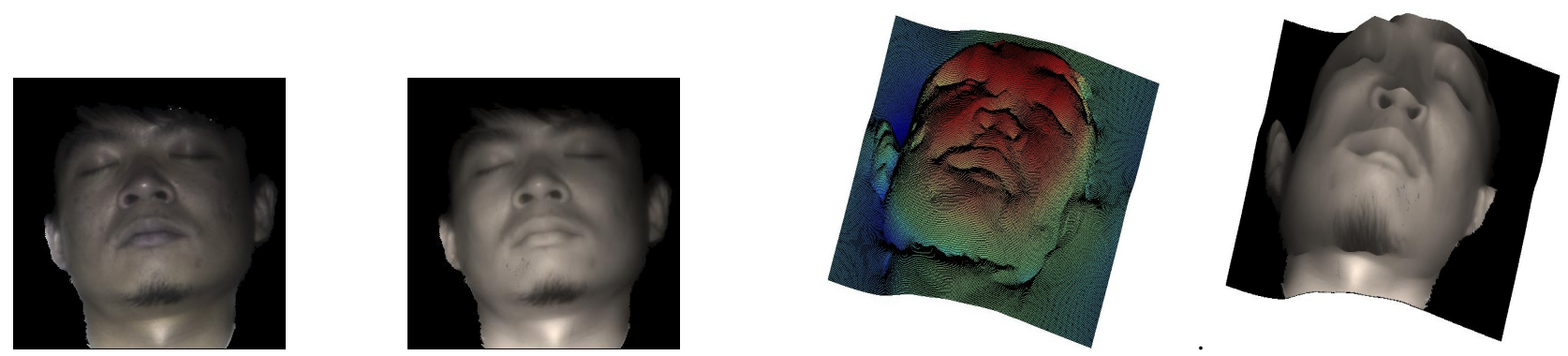

Figure 5. Results for a real world image. From left to right: The input image; Estimated image rendered with the recovered surface normals and albedo; Depth map; Result of texture-mapping the image in the second column onto the depth map in the third column.

\section{Experiments}

We now present results on the recovery of diffuse reflectance $\rho$ and the shape from a single input image. To this end, we have performed experiments on synthetic and realworld imagery. Our synthetic data is given by the image of a candle stick rendered using the reflectance model in Equation (1). Our real-world data is comprised by the imagery for 51 human subjects, each captured under an incandescent light positioned frontally with respect to the subjects under study. All our imagery is given by 30 bands in the range $430 \mathrm{~nm}-720 \mathrm{~nm}$, in $10 \mathrm{~nm}$ steps.

We first turn our attention to the candle stick image. Our candle stick is made of two different materials and, therefore, can be divided into two regions, which we name k1 and k2. We use two different albedos acquired in house with an spectrometer for rendering purposes. The first of these is that of Aloevera leaf $(\mathrm{k} 1)$ and yellow paper $(\mathrm{k} 2)$. We also use two indeces of refraction. These correspond to Silicon (Si) for k1 and BK7 for k2. In the left-hand panel of Figure 2, we show the candle stick image taken as input by our algorithm. In the middle panel we show that which has been rendered making use of the estimated albedos $\rho$ and surface normals $\vec{N}$. In the right-hand panel, we show the estimated surface depth after the application of the Frankott and Chellappa integration method.

In Figure 3, we turn our attention to the albedo recov- ered by our method. In the figure, we show, in the top row, the reflectance for the Aloevera leaf and the yellow paper as used for rendering the input image. The bottom row shows the average albedo recovered by our method for the pixels whose reflectance corresponds to the spectra in the top row. In the panels, the error-bars correspond to the standard deviation of the pixel spectra. Note that our recovered albedo is in close accordance with the reflectance used for rendering the input image. Moreover, the standard deviation is low, which implies that our method can reliably recover the reflectance without being overly affected by shading and specularities.

We now turn our attention to the real world imagery. In Figure 4 and 5, we show the results yielded by our method for two sample subjects. From left-to-right, the figure shows the input image, the image rendered using the recovered albedo and surface normals, the face depth-map after the application of the method of Frankott and Chellappa and the relief obtained when the image on the second panel is texture-mapped onto the depth map on the third column. Note that the face shape for the two subjects has been captured by the surface normals, which, in turn, yields surface depth maps in good accordance with the expected 3D shape. In Figure 6, we show the average skin reflectance obtained by our method for the three main ethnicities in the dataset (Caucasian, Asian and oriental skin). Our results are consis- 


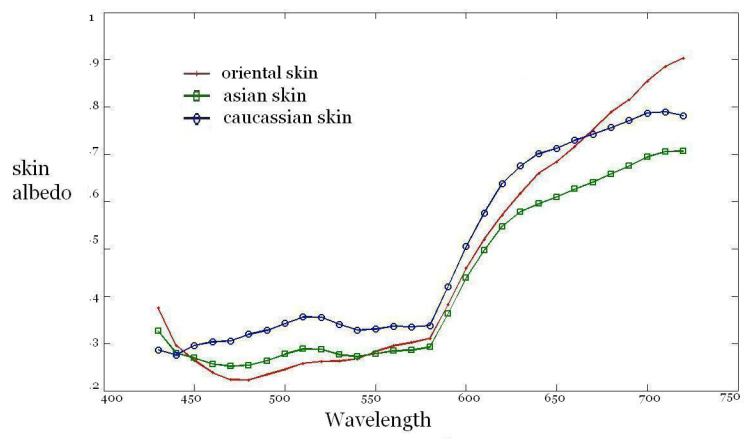

Figure 6. Estimated skin reflectance for the three skin types

tent with the real-world observation regarding darker skin types in the sense that these should reflect a smaller proportion of incident light in the $450 \mathrm{~nm}-550 \mathrm{~nm}$ range. Furthermore, all plots exhibit a gradual increase with respect to wavelength with a dip around $575 \mathrm{~nm}$. This is in accordance with the results reported in [1].

\section{Conclusions}

In this paper, we have presented a method that recovers surface shape and reflectance from a single hyperspectral image. The method employs a linear combination of the Torrance-Sparrow, Beckmann-Kirchhoff and Wolff models so as to model the specular lobe, specular spike, and diffuse reflection. This permits us to recover the reflectance and the surface normals via an iterative optimisation approach. With the normals in hand, we recover the surface depth making use of the method in [8]. We have illustrated the utility of our method for both synthetic and real world imagery.

\section{References}

[1] Elli Angelopoulou, Rana Molana, and Kostas Daniilidis. Multispectral skin color modeling. In IEEE conference on Computer Vision and Pattern Recognition (CVPR, pages 635-642, 2001. 6

[2] P. Beckmann and A. Spizzichino. The Scattering of Electromagnetic Waves from Rough Surfaces. Pergamon Press, 1963. 2

[3] Peter N. Belhumeur and David J. Kriegman. What is the set of images of an object under all possible lighting conditions. IJCV, 28:270-277, 1998. 1

[4] M. Bichsel and A.P. Pentland. A simple algorithm for shape from shading. In IEEE Proceedings of Computer Vision and Pattern Recognition, pages 459-465, 1992. 2
[5] R. L. Cook and K. E. Torrance. A reflectance model for computer graphics. volume 15, pages 307-316, 1981. 2

[6] Ron O. Dror, Edward H. Adelson, and Alan S. Willsky. Recognition of surface reflectance properties from a single image under unknown real-world illumination, 2001. 1

[7] P. Dupuis and J. Oliensis. Direct method for reconstructing shape from shading. In IEEE Proceedings of Computer Vision and Pattern Recognition, pages 453458, 1992. 2

[8] R. T. Frankot and R. Chellappa. A method for enforcing integrability in shape from shading algorithms. IEEE Transactions on Pattern Analysis and Machine Intelligence, 10:439-451, 1988. 1, 2, 4, 6

[9] B. K. P. Horn. Shape from shading: A method for obtaining the shape of a smooth opaque object from one view. In MIT AI-TR, 1970. 1

[10] B. K. P. Horn. Height and gradient from shading. International Journal of Computer Vision, 5(1):37-75, 1990. 1

[11] B. K. P. Horn and M. J. Brooks. The variational approach to shape from shading. CVGIP, 33(2):174208, 1986. 1

[12] Cong Phuoc Huynh and Antonio Robles-Kelly. Simultaneous photometric invariance and shape recovery. 2009 IEEE 12th International Conference on Computer Vision, pages 1757-1764, 2009. 4

[13] Gudrun J. Klinker, Steven A. Shafer, and Takeo Kanade. A physical approach to color image understanding. International Journal of Computer Vision, 4:7-38, 1990. 1

[14] J. L. Krakauer. Computer analysis of visual properties of curved objects. In Massachusetts Institute of Technology, AI-TR, 1971. 1

[15] J. H. Lambert. The Photometria, sive, De mensura et gradibus luminis, colorum et umbrae. Eberhard Klett, Augsburg, 1760. 3

[16] Y. G. Leclerc and A. F. Bobick. The direct computation of height from shading. In Proceedings of Computer Vision and Pattern Recognition, pages 552-558, 1991. 2

[17] K. Levenberg. A method for the solution of certain problems in least squares. Quart. Appl. Math., 2:164$168,1944.3$ 
[18] R. R. Lewis. Making shaders more physically plausible. In Proceedings of the Fourth Eurographics Workshop on Rendering, pages 47-62, 1993. 2

[19] Stephen Lin and Heung-Yeung Shum. Separation of diffuse and specular reflection in color images. Computer Vision and Pattern Recognition, IEEE Computer Society Conference on, 1:341, 2001. 1

[20] D. Marquardt. An algorithm for least-squares estimation of nonlinear parameters. SIAM J. Appl. Math., 11:431-441, 1963. 3

[21] S.G. Narasimhan, V. Ramesh, and S.K. Nayar. A Class of Photometric Invariants : Separating Material from Shape and Illumination. In IEEE International Conference on Computer Vision (ICCV), volume 2, pages 1387-1394, Oct 2003. 1

[22] S. K. Nayar and M. Oren. Visual appearance of matte surfaces. SCIENCE, 267:1153-1156, 1995. 2

[23] Shree K. Nayar and Ruud M. Bolle. Reflectance based object recognition, 1996. 1

[24] S.K. Nayar, K.Ikeuchi, and T. Kanade. Surface Reflection: Physical and Geometrical Perspectives. IEEE Transactions on Pattern Analysis and Machine Intelligence, 13(7):611-634, Jul 1991. 2, 3

[25] B. T. Phong. Illumination for computer generated pictures. Communications of the ACM, 18(6):311-317, 1975. 2

[26] T. Rindfleisch. Photometric method for lunar topography. Photogrammetric Engineering, 32(2):262-277, 1966. 1

[27] C. Schlick. A fast alternative to phong's specular model. Graphics Gems, 4:363-366, 1994. 2

[28] C. Schlick. A survey of shading and reflectance models. Computer Graphics Forum, 13(2):121-131, 94. 2

[29] Cordelia Schmid and Roger Mohr. Local grayvalue invariants for image retrieval. IEEE Transactions on Pattern Analysis and Machine Intelligence, 19:530535, 1997. 1

[30] K. E. Torrance and E. M. Sparrow. Theory for offspecular reflection from roughened surfaces. Journal of the Optical Society of America, 57:1105-1114, 1967. 2

[31] T. Wei and R. Klette. Theoretical analysis of finite difference algorithms for linear shape from shading. In $C A I P$, pages 638-645, 2001. 2
[32] L. B. Wolff. Diffuse-reflectance model for smooth dielectric surfaces. Journal of the Optical Society of America, pages 2956-2968, 1994. 3

[33] L. B. Wolff. On the relative brightness of specular and diffuse reflection. In Int. Conf. on Comp. Vision and Patt. Recognition, pages 369-376, 1994. 2

[34] R. J. Woodham. Analysing images of curved surfaces. Artificial Intelligence, (17):117-140, 1981. 1

[35] E.R. Worthington, P.L. Hancock. New constraints on data-closeness and needle map consistency for shapefrom-shading. EEE Transactions on Pattern Analysis and Machine Intelligence, 21:1250-1267, 1999. 4

[36] Z. Wu and L. Li. A line-integration based method for depth recovery from surface normals. CVGIP, 43(1):53-66, July 1988. 2

[37] R. Zhang, P. S. Tsai, J. E. Cryer, and M. Shah. Shape from shading: A survery. IEEE Trans. on Pattern Analysis and Machine Intelligence, 21(8):690-706, 1999. 2 